\title{
JNNP
}

\section{Evaluation of tumour response after gamma knife radiosurgery for residual vestibular schwannomas based on MRI morphological features}

S-Y Yang, D G Kim, H-T Chung, et al.

J Neurol Neurosurg Psychiatry 2008 79: 431-436 originally published online August 2, 2007

doi: 10.1136/jnnp.2007.119602

Updated information and services can be found at:

http://jnnp.bmj.com/content/79/4/431.full.html

These include:

References This article cites 23 articles, 2 of which can be accessed free at: http://jnnp.bmj.com/content/79/4/431.full.html\#ref-list-1

Email alerting Receive free email alerts when new articles cite this article. Sign up in the service box at the top right corner of the online article.

Topic collections Articles on similar topics can be found in the following collections

Neuromuscular disease (8309 articles)

Neurooncology (1036 articles)

Peripheral nerve disease (3607 articles)

Radiology (14701 articles)

Notes

To order reprints of this article go to:

http://jnnp.bmj.com/cgi/reprintform

To subscribe to Journal of Neurology, Neurosurgery \& Psychiatry go to:

http://jnnp.bmj.com/subscriptions 


\title{
Evaluation of tumour response after gamma knife radiosurgery for residual vestibular schwannomas based on MRI morphological features
}

\author{
S-Y Yang, ${ }^{1,2}$ D G Kim, ${ }^{2,3}$ H-T Chung, ${ }^{2,3}$ S-H Park, ${ }^{2,4}$ S H Paek, ${ }^{2,3}$ H-W Jung ${ }^{2,3}$
}

${ }^{1}$ Department of Neurosurgery, DongGuk University International Hospital, Goyang, Gyeonggi-do, Republic of Korea; ${ }^{2}$ Clinical Research Institute, Seoul National University Hospital, Seoul, Republic of Korea;

${ }^{3}$ Department of Neurosurgery, Seoul National University College of Medicine, Seoul, Republic of Korea; ${ }^{\prime}$ Department of Pathology, Seoul National University College of Medicine, Seoul, Republic of Korea

Correspondence to: Dong Gyu Kim, MD, PhD, Professor, Department of Neurosurgery, Seoul National University College of Medicine, 28 Yeongon-dong, Jongno-gu, Seoul 110-744, Korea; gknife@ plaza.snu.ac.kr

Received 4 March 2007 Revised 15 May 2007 Accepted 7 July 2007

Published Online First

2 August 2007

\section{ABSTRACT}

Objective: To evaluate tumour response after gamma knife (GK) radiosurgery for residual vestibular schwannoma (VS) based on MRI morphological features.

Methods: Sixty-one patients with histopathologically confirmed VS underwent GK radiosurgery with marginal tumour doses of 9.0-14.0 Gy (mean, 12.5). Mean tumour volume at GK radiosurgery was $3.65 \mathrm{ml}$ (range, 0.5215.50). GK radiosurgery was performed 0.3-95.7 months (median, 5.8) after microsurgery. Tumour volumes and half-reduction time were calculated using serial MRI. The morphological features of VS were documented by premicrosurgical MRI. Histopathological investigation included Antoni-type dominance, the proliferation marker Ki-67 and tumour vascularity.

Results: Median duration of radiological follow-up was 53.7 months (range, 24.1-102.2) and the 8-year actuarial tumour control rate was $93.5 \%$. No factor was associated with tumour control, although a cystic VS had borderline significance $(p=0.089)$. Mean tumour half-reduction time was 8.70 years (range, 0.57-79.89) and tumour half-reduction time in cystic VS proved to be significantly shorter than those in solid VS $(p=0.006)$. Thrombotic vessels ( $p=0.015$ ) and abnormal vessel proliferation $(p=0.003)$ were significantly more prominent in cystic VS than those in solid VS.

Conclusions: GK radiosurgery appeared to be an effective treatment modality for residual tumour control after microsurgery. Owing to having relatively abundant tumour vascularity, residual solid portions of cystic VS resulted in efficient shrinkage after GK radiosurgery. Therefore, GK radiosurgery was found to be a rewarding therapeutic approach to the residual solid portions of cystic VS.

Numerous investigators have reported that gamma knife (GK) radiosurgery is a safe and effective treatment for vestibular schwannoma (VS). ${ }^{12}$ This has encouraged GK radiosurgery to be adapted as an important treatment option for VS. In an effort to improve radiosurgical results, several groups ${ }^{1-6}$ have analysed their experiences in efforts to identify factors associated with radiosurgical failure. Their studies illustrate a number of treatment failures associated with the following factors: targeting error, larger tumour volume and cystic type. Although much has been learned from these studies, no study has been conducted to determine whether the morphological features based on MRI are associated with GK radiosurgical response. To address this issue, we analysed treatment outcomes and tumour halfreduction time after GK radiosurgery of histopathologically proven VS based on clinical and MRI morphological features. In addition, the reason why each tumour response was different depending on MRI morphological features was explained on the basis of histopathological appearance.

\section{PATIENTS AND METHODS}

\section{Patient characteristics}

Between January 1998 and December 2004, 280 patients with unilateral VS underwent GK radiosurgery at our hospital. Because tumours in some patients increased in size slightly after radiosurgery, but were subsequently either stabilised or regressed, ${ }^{27}$ patients with less than a 24 -month period after radiosurgery (from January 2005 to December 2006) were excluded. Patients associated with pre- or post-microsurgical radiotherapy, or neurofibromatosis, were also excluded. Of these 280 patients, 61 had previously undergone incomplete microsurgical tumour removal. Premicrosurgical mean tumour volume was $20.6 \pm 11.1 \mathrm{ml}$ (range, 4.1-44.5). There were 41 women and 20 men whose median age at the time of radiosurgery was 41 years (range 18-67).

Histopathologically, they had all been diagnosed as having VS. The extent of internal debulking depended on the findings of a facial nerve electrophysiological monitoring system (NIM nerve integrity monitoring system; Medtronic Xomed Surgical Products, Inc., Jacksonville, FL) and on the surgeon's sense. Dissection of the tumour was performed between two arachnoid membranes of the tumour. Incomplete microsurgical removal of tumour shells or capsules adhering to the neural structures with tenacity was attempted to ensure a good functional outcome of the cranial nerves and brain stem. However, all cystic portions of tumour were intentionally removed during an operation for adjuvant GK radiosurgery, if possible. The tumour cells of the cyst wall strongly and diffusely expressed S-100 protein by immunohistochemistry.

The institutional review board of our hospital approved this study. However, it did not require informed consent from these patients for their inclusion in this study because the study depended only on information obtained as part of routine clinical care and patient medical records. A careful review of medical records, histopathological records and radiological images was conducted retrospectively.

Morphological features of vestibular schwannoma The morphological features of VS were determined by an extensive analysis of all imaging data by two 
observers. Where differences between the observers' opinions were encountered, the imaging data were re-examined by both observers and results were recorded after an agreement was reached. Morphological features were assessed using premicrosurgical MRI in terms of: the presence of intratumoral necrosis, intratumoral haemorrhage, enhancement pattern, tumour nature and tumour contour (fig 1). Based on our recent study, ${ }^{8}$ intratumoral haemorrhage was defined as an intratumoral fluid-fluid level and/or a dark signal suggesting hemosiderin deposits on T2-weighted images. Cystic VS was defined as a tumour with enhanced cysts making up at least one-third of the tumour volume on gadolinium-enhanced T1-weighted images. All cases underwent MRI on a 1.5-tesla system, which included $2 \mathrm{~mm}$ axial non-enhanced T1-weighted images, $2 \mathrm{~mm}$ axial T2-weighted images, $1 \mathrm{~mm}$ axial three-dimensional constructive interference in steady-state images and $2 \mathrm{~mm}$ axial gadolinium-enhanced T1-weighted three-dimensional gradientecho images $(0.1 \mathrm{mg} / \mathrm{kg}$ body weight). MRI morphological characteristics of VS are shown in figure 1 and are summarised in table 1.

\section{Histopathological investigation}

Histopathological investigation included Antoni-type dominance, the proliferation marker Ki-67 and tumour vascularity. Antoni-type dominance was determined if there was an obvious predominance of Antoni-type A or B tissue after examination of all slides for an individual tumour. If there was no obvious predominance, the tumour was categorised as a mixed type. To quantify the percentage of nuclei staining with Ki-67, a nuclear antigen, at least 500 tumour cells were manually counted three times in the region of tumour with the highest nuclear staining, and an average percentage of positively stained nuclei was calculated. Abnormal vessel proliferation and thrombotic vessels were investigated to evaluate the tumour vascularity.

\section{Radiosurgical technique}

Patients were treated using a GK B \& C-model (Elekta, Stockholm, Sweden) with Leksell Gamma Plan (Elekta). T1weighted three-dimensional gradient-echo MRI scans were obtained before and after gadolinium enhancement to determine the target. Treatment planning was performed on axial images supplemented with coronal and sagittal reconstructed images. In the early days of our experience with GK, we tried to treat larger VS (more than $14 \mathrm{ml}$ ). We reduced the prescription dose to minimise radiation-induced adverse effects. However, most of the large tumours showed severe tumour swelling after GK surgery, causing symptoms such as severe headache, vomiting and dizziness even at these lowered prescription doses. Since then, we have not treated larger VS with GK. Early in this series, marginal doses $\leqslant 11$ Gy were prescribed only for large tumours, whereas later in this series, 12-13 Gy became the usual marginal dose for most patients. The current dose prescription scheme is to give 12 Gy when there is serviceable hearing or $13 \mathrm{~Gy}$, otherwise, which is compatible with recent trends.

There were occasional treatment failures because of cyst expansion in cystic VS after radiosurgery. ${ }^{5}$ Therefore, we treated our series of cystic VS in the following manner: The small-sized cystic intracanalicular tumours were managed conservatively by means of biannual MRI and audiometry, whereas the large cystic tumours were treated by a combination of microsurgery and adjuvant GK radiosurgery. Moreover, to obtain a better radiosurgical outcome, if possible, the cystic portion was removed, or cysts were partially removed or ruptured during the operation in the cases described above.

Marginal tumour doses varied from 9.0 to 14.0 Gy (mean, 12.5 Gy). Marginal tumour doses were 9.0 Gy $(n=1), 10.5$ Gy $(n=2), 11.0$ Gy $(n=3), 12.0$ Gy $(n=20), 13.0$ Gy $(n=30), 13.5$ Gy $(n=3)$ or 14.0 Gy $(n=2)$. The maximum dose varied from 18.0 to 28.0 Gy (mean, 24.9 Gy). The marginal dose was prescribed to the $50 \%$ isodose line in 58 patients, $49 \%$ in 1 and $52 \%$ in 2 patients. The number of isocentres treated per patient varied from 3 to 21 (median, 13 isocentres).

GK radiosurgery right after the operation made it difficult to discriminate between postoperative change and residual tumour, and to plan for the radiosurgical treatment due to insufficient shrinkage of the gutted tumour. Years of experience have taught us that gutted tumours were usually shrunk about 4-6 months later in follow-up MRI, which was supposed to be the right point of the GK radiosurgery. For this reason, since 2000, GK radiosurgery has been performed 4-6 months after the microsurgery. The interval between microsurgery and GK radiosurgery ranged from 0.3 to 95.7 months (median, 5.8). The mean tumour volume at GK radiosurgery was $3.65 \mathrm{ml}$ (range, 0.52-15.50).

\section{Follow-up evaluations}

Each patient was clinically followed up at 1, 3, 6, 12, 18 and 24 months after GK radiosurgery and then annually. Clinical assessments, including a neurological examination and audiometry, were performed every 6 months after GK radiosurgery for the first 2 years and then annually using an individually designed protocol. Audiometry results were evaluated according to the Gardner-Robertson (G-R) grade. ${ }^{9}$ Serviceable hearing was defined as G-R grade I or II. Before microsurgery, 10 patients had a serviceable hearing but 51 did not. Serviceable hearing was preserved in 5 of these 10 patients at the time of GK radiosurgery. Facial nerve function was assessed based on the House-Brackmann (H-B) grading system. ${ }^{10}$ Functional preservation of the facial nerve was defined if postoperative $\mathrm{H}-\mathrm{B}$ grade was either 1 or 2 . Only four patients had preoperative mild facial nerve palsy ( $\mathrm{H}-\mathrm{B}$ grade 2 ), whereas the other 57 exhibited normal facial expressions. After microsurgery, facial function was normal (grade 1) in 52 patients (85\%), but was classified as grade 2 in 6 patients (10\%), grade 4 in $2(3 \%)$ and grade 5 in 1 $(2 \%)$ at the time of GK radiosurgery. MRI was normally conducted at 3, 6 and 12 months after GK radiosurgery, at 1-year intervals for the next 2 years, and biennially thereafter.

Tumour control was assessed from two points of view-that is, radiographic control and clinical control. Radiographic tumour progression was strictly defined as any temporary or sustained increase in tumour diameter of at least $1 \mathrm{~mm}$ in two dimensions or $2 \mathrm{~mm}$ in any direction. Because tumours in some patients slightly increased in size after radiosurgery, but subsequently either stabilised or regressed, ${ }^{2}{ }^{7}$ clinical treatment failure was defined as a requirement for salvage treatment due to GK radiosurgery-related complications or tumour enlargement. For these reasons, we describe tumour control as neither radiographic tumour progression nor requirement for salvage treatment.

\section{Calculation of tumour volume and half-reduction time}

Tumour control rate after GK radiosurgery in VS is well known to be high. Definition of tumour control, like ours, includes complete remission, partial remission, stable and sustained increase in tumour diameter of at least $1 \mathrm{~mm}$ in two dimensions 

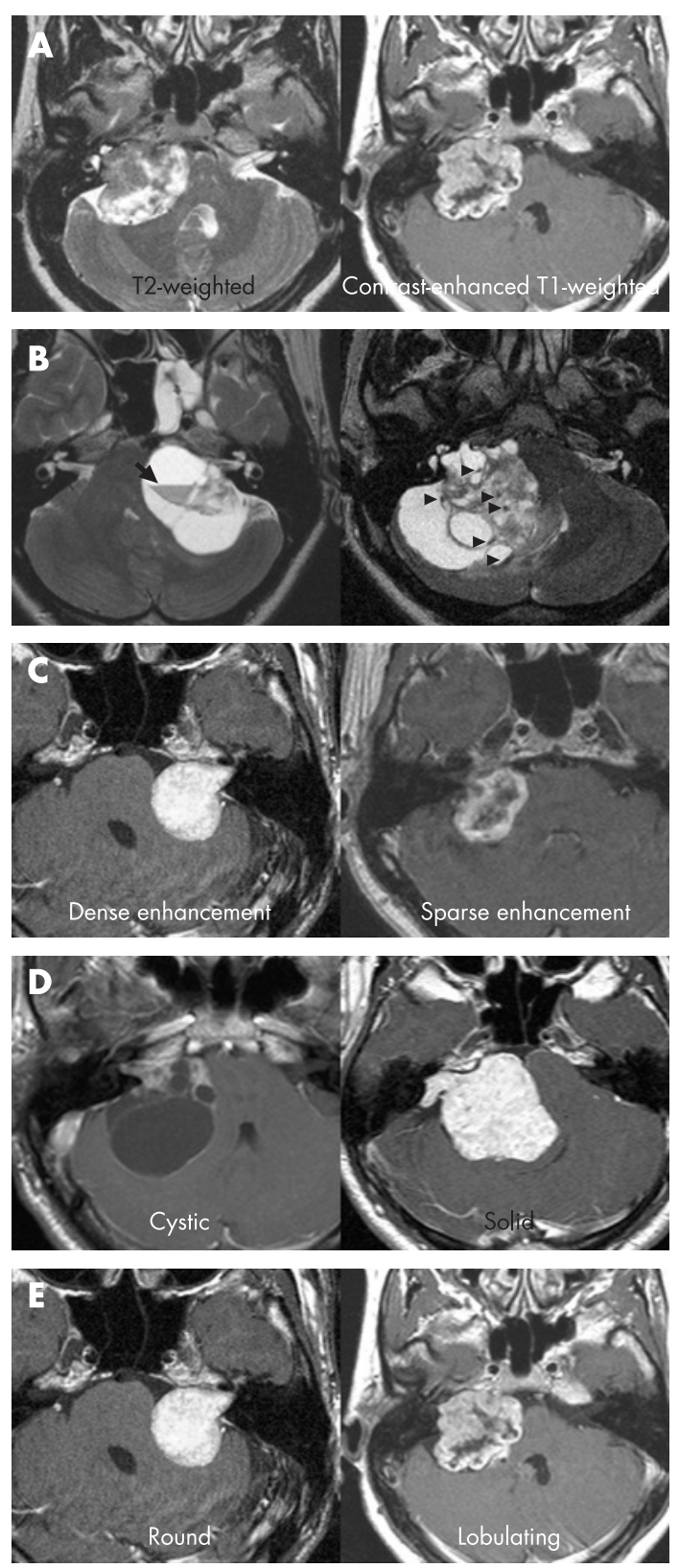

Figure 1 Morphological features of vestibular schwannoma (VS) based on MRI appearance. Intratumoral necrosis was defined as central homogeneous high signal intensity on T2-weighted images and defective central enhancement after gadolinium injection (A). Intratumoral haemorrhage was defined as an intratumoral fluid-fluid level and/or a dark signal suggesting hemosiderin deposits on T2-weighted images (arrows point to fluid-fluid level, arrowheads to dark signals showing conspicuous hemosiderin deposits) (B). Enhancement pattern (dense or sparse) (C). Tumour nature (cystic or solid); cystic VS was defined as a tumour with enhanced cysts making up at least one-third of the tumour volume on gadolinium-enhanced T1-weighted images (D). Tumour contour (round or lobulating) (E).

or $2 \mathrm{~mm}$ in any direction. So, tumour control rate did not reflect a subtle difference in radiosurgical responses based on morphological features of the tumour. For this reason, tumour halfreduction time was adopted to obtain a subtle difference in radiosurgical responses based on morphological features of the tumour. Namely, tumour half-reduction time was used to find how well the tumour responds to the radiation rather than how well it is controlled by the radiation.
Table 1 Morphological characteristics of vestibular schwannoma in $\mathrm{MRI}$ at diagnosis

\begin{tabular}{ll}
\hline Characteristics & Variables \\
\hline $\begin{array}{l}\text { Volume at diagnosis, ml } \\
\quad \text { Mean }\end{array}$ & $20.6 \pm 11.1$ (range, 4.1-44.5) \\
Intratumoral necrosis, no of patients (\%) & $23(37.7)$ \\
$\quad$ Yes & $38(62.3)$ \\
No & $19(31.1)$ \\
Intratumoral hemorrhage, no of patients (\%) & $42(68.9)$ \\
$\quad$ Yes & \\
$\quad$ No & $37(60.6)$ \\
Enhancement pattern, no of patients (\%) & $24(39.4)$ \\
$\quad$ Dense & \\
$\quad$ Sparse & $25(41.0)$ \\
Tumour nature, no of patients (\%) & $36(59.0)$ \\
$\quad$ Cystic & \\
$\quad$ Solid & $44(72.1)$ \\
Tumour contour, no of patients (\%) & $17(27.9)$ \\
$\quad$ Round \\
$\quad$ Lobulating
\end{tabular}

Tumour volumes were measured in MRI using the Osiris program (version 4.0; Digital Imaging Unit/University Hospital of Geneva, Geneva, Switzerland) or Leksell Gamma Plan (Elekta Ltd.). Tumour volumes were computed by specifying an object in a series of regions of interest (ROI) over a set of multi-planar images and then summing the ROI areas in each plane. MRI stored in host computers were retrieved and connected to a Picture Archiving and Communication System, and then electronically transferred as a DICOM file to determine tumour volumes using the Osiris program. The standard deviation associated with repeated measurements of tumour volume from the same MRI was within $\pm 1.5 \%$. Individual sequential tumour volume plotting during the follow-up period revealed that the changes in tumour volumes after GK radiosurgery followed a decreasing exponential pattern. For this reason, to identify radiosurgical effects, tumour half-reduction time was calculated using natural logarithms (fig 2). $V_{0}$ and $T_{0}$ are tumour volume and date at the time of GK radiosurgery, and $\mathrm{V}_{\mathrm{A}}$ and $\mathrm{T}_{\mathrm{A}}$ are tumour volume and date of the last follow-up.

$\mathrm{V}_{\mathrm{A}}=\mathrm{V}_{0} \exp \left[-\lambda\left(\mathrm{T}_{\mathrm{A}}-\mathrm{T}_{0}\right)\right]$

Then,

$\lambda=\left(\log \mathrm{V}_{0}-\log \mathrm{V}_{\mathrm{A}}\right) /\left(\mathrm{T}_{\mathrm{A}}-\mathrm{T}_{0}\right)$

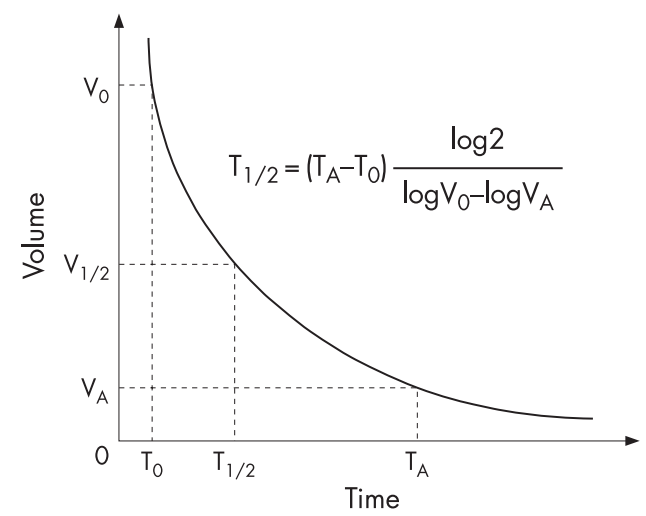

Figure 2 Formula used for determining tumour half-reduction time: $V_{0}$ and $T_{0}$ are the tumour volume and date of gamma knife radiosurgery, and $\mathrm{V}_{\mathrm{A}}$ and $\mathrm{T}_{\mathrm{A}}$ are the tumour volume and date of the last follow-up. 
On the other hand, if we set time ' $T^{\prime}$ as when the tumour volume is reduced to one-half of the initial volume, then

$\mathrm{V}^{\prime}=\mathrm{V}_{0} / 2=\mathrm{V}_{0} \exp \left[-\lambda\left(\mathrm{T}^{\prime}-\mathrm{T}_{0}\right)\right]$

Then, the tumour half-reduction time $\left(T_{1 / 2}\right)$ is given as

$\mathrm{T}_{1 / 2}=\mathrm{T}^{\prime}-\mathrm{T}_{0}=(\log 2) / \lambda=\left(\mathrm{T}_{\mathrm{A}}-\mathrm{T}_{0}\right) /\left[(\log 2) /\left(\log \mathrm{V}_{0}-\right.\right.$ $\left.\left.\log \mathrm{V}_{\mathrm{A}}\right)\right]=\left(\left[\mathrm{T}_{\mathrm{A}}-\mathrm{T}_{0}\right] \times \log 2\right) /\left(\log \mathrm{V}_{0}-\log \mathrm{V}_{\mathrm{A}}\right)$

\section{Statistical analysis}

Statistical analyses were performed using SPSS version 12.0. Tumour control rates were calculated using the KaplanMeier method. Factors affecting tumour control were assessed using the log-rank test and the Cox proportional hazards model. A final multivariate analysis was performed using stepwise backward elimination. The Mann-Whitney test was used to determine the significance of tumour half-lives between groups classified by the presence or absence of each clinical factor. The different valuables-that is, sex, age, tumour volume and marginal dose-were compared using the chisquare test and the Mann-Whitney test between groups classified by the presence or absence of each clinical factor. For multivariate analysis, we used a logistic regression model to analyse correlations between tumour half-reduction time and clinical factors. Continuous variables were dichotomised based on their median values and clinical significance. $p$ Values of less than 0.05 were considered to be statistically significant.

\section{RESULTS}

Median radiological follow-up period after GK radiosurgery was 53.7 months (range, 24.1-102.2). All patients remained alive, and no patient had experienced a transient or permanent neurological deficit at final follow-up or been treated with steroids for complications related to GK radiosurgery.

\section{Tumour control after GK radiosurgery}

At the last follow-up MRI scans, 50 of the 61 patients had a smaller tumour volume and eight a slightly larger $(<1 \mathrm{~mm}$ in two dimensions or $<2 \mathrm{~mm}$ in any direction) tumour volume. The remaining three with solid VS experienced radiographic tumour progression within 4 years of GK radiosurgery; one patient was re-treated using GK radiosurgery but the others were observed only because of no symptoms. The 4- and 8-year actuarial tumour control rates were $96.5 \pm 2.4 \%$ and $93.5 \pm 3.7 \%$, respectively (fig 3 ). No factor was associated with tumour control, although a cystic VS had borderline favourable significance $(p=0.089)$.

\section{Clinical response after GK radiosurgery}

Neurological evaluations showed that no worsened KPS score occurred after GK radiosurgery. Facial nerve function was eventually preserved in 58 (grade 1 in 52 patients, grade 2 in 6 , grade 4 in 2 , and grade 5 in 1) of 61 patients (95\%) who could be evaluated, and no additional deterioration of facial nerve function after GK radiosurgery was observed at last follow-up.

During the follow-up period, 3 of the 5 patients who had serviceable hearing at the time of GK radiosurgery had preserved serviceable hearing and 2 retained their preradiosurgical G-R grades at last follow-up. Serviceable hearing was eventually preserved in 30\% (3/10) of patients at last follow-up. No acute or chronic toxicity induced by GK radiosurgery was detected during the follow-up.

\section{Tumour half-reduction time after GK radiosurgery}

In addition to the three patients who experienced treatment failure, $18 \%(11 / 61)$ of patients were identified to have temporary or permanent tumour diameter increases of $2 \mathrm{~mm}$ and below. In three patients of these eleven, the tumours shrank to less than the size measured at GK radiosurgery, whereas in the other eight patients the tumours remained stable in size. Temporary tumour swelling was found 3-10 months after GK radiosurgery and spontaneously regressed without treatment. Patients were dichotomised based on tumour half-reduction time after GK radiosurgery, as follows: a negative value (not radiographic treatment failure but tumour growth) for 11 tumours (18\%) and a positive value (tumour shrinkage) for 50 (82\%). The negative group consisted of 10 solid VS and 1 cystic VS. The negative value for a solid VS was significantly more frequent than that for cystic VS $(p=0.020)$. The whole negative value group was excluded from the tumour half-reduction time analysis. Tumour half-reduction times after GK radiosurgery ranged from 0.57 to 79.89 years, with a mean of 8.70 years. The univariate analysis showed associations both between the tumour half-reduction time and cystic VS $(p=0.009)$, and between the tumour half-reduction time and intratumoral haemorrhage $(p=0.042)$. In multivariate analysis, cystic VS was only identified as an independent factor for tumour halfreduction time $(p=0.006)$. However, the intratumoral haemorrhage failed to reach significance $(p=0.057)$. Mean tumour halfreduction times for cystic VS and solid VS were 2.58 years and 14.34 years, respectively (table 2). There was no statistically significant difference between the volumes at GK radiosurgery in the cystic VS group (mean $3.58 \mathrm{ml}$ ) and the volumes at GK radiosurgery (mean $3.69 \mathrm{ml}$ ) in the solid VS group $(p=0.825)$. The marginal doses in patients with cystic VS (mean 12.6 Gy) did not differ from that in patients with solid VS (mean 12.4 Gy) $(p=0.406)$. The maximum doses were not significantly different between patients with cystic VS (mean 25.2 Gy) and those with solid VS (mean 24.9 Gy) ( $p=0.379$ ). Also, there was no statistically significant difference in the other baseline characteristics (sex and age) between patients with cystic VS and those with solid VS.

Histopathologically, no significant differences were found between the cystic VS group and the solid VS group as to Antoni-type dominance and the Ki-67 proliferative index ( $p=0.462$ and $p=0.730$, respectively). However, thrombotic vessels $(p=0.015)$ and abnormal vessel proliferation $(p=0.009)$ were significantly more prominent in cystic VS than those in solid VS. Also, thrombotic vessels and abnormal vessel proliferation were associated with intratumoral haemorrhage $(p=0.033$ and $p=0.025$, respectively), but Antoni-type dominance and the Ki-67 proliferative index were not $(p=0.715$ and $p=0.598$, respectively). On the other hand, histopathological characteristics of the other three groups' morphological features (presence of intratumoral necrosis, enhancement pattern and tumour contour) based on MRI showed no statistical differences.

The factors investigated affecting tumour half-reduction time are summarised in table 2.

\section{DISCUSSION}

Unlike other studies, ${ }^{5}{ }^{6}$ the tumour control rate of cystic VS was found to be higher than that of solid VS after GK radiosurgery in our study. Also, cystic VS had a much shorter mean tumour half-reduction time than solid VS. The suggested mechanisms for these results are possibly explained by being related to two major radiobiological effects based on histopathological characteristics. The major radiobiological effect of GK radiosurgery 


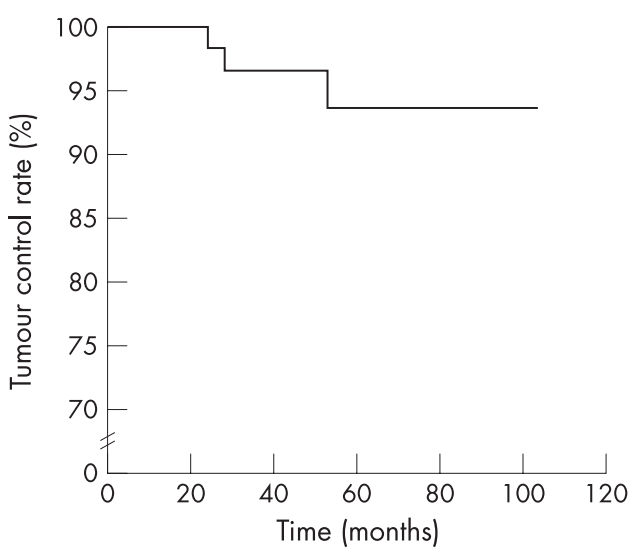

Figure 3 Graph demonstrating a Kaplan-Meier curve of tumour control rates in 61 patients with vestibular schwannoma treated using gamma knife radiosurgery with microsurgery.

is a combination of delayed vascular and cytotoxic effects. ${ }^{11}{ }^{12}$ First, delayed vascular effects result from radiation-induced damage to tumour nutrient vessels. The importance of the vasculature in radiation-induced tumour necrosis is well known; one popular hypothesis regarding pathogenesis after GK radiosurgery is that damage to capillary endothelium ultimately interrupts blood flow and results in secondary ischemic tumour cell death. ${ }^{11} 1314$ In addition, the abnormal vessels of neoplasms are more sensitive to radiosurgery than normal surrounding vessels. ${ }^{11}{ }^{15}$ It has been observed that several of the histopathological characteristics of cystic VS are different from those of solid VS. Hypervascularity and presence of Antoni-type B tissue area in cystic VS are more common findings than those in solid VS. $^{81618}$ Moreover, abnormal sinusoid or telangiectasis-like vessels, thrombotic vessels and abnormal vessel proliferation for cystic VS are more prominent than those for solid VS. ${ }^{8}{ }^{16}$ The solid portions of cystic VS have been reported to be more vascular than those of solid VS. ${ }^{19}{ }^{20}$ In common with other studies, our study showed that abnormal vessel proliferation and thrombotic vessels in cystic VS were more commonly found than those in solid VS. From the perspective of delayed vascular effects, cystic VS with cystic portion removal may show more effectiveness in GK radiosurgery than solid VS.

Second, the cytotoxic effect is associated with the DNA damage that occurs secondary to the direct effects of gamma rays, and is due to the strand breaks from the oxygen radicals generated. From the perspective of cytotoxic effect, ultimate tumour control after irradiation may be determined by a number of tumour cells. ${ }^{21}$ The smaller number of tumour cells in cystic VS, which are known to be composed of Antoni-type B tissue rather than A tissue compared with those in solid VS of the same size, may contribute to better tumour control of cystic VS. ${ }^{18} 2122$ However, we found no relationship between cystic VS and the distribution of Antoni-type B tissue.

Based on these theoretical backgrounds and our results, abnormal tumour vessel proliferation, relative hypervascularity and the abundance of Antoni-type B tissue in the solid portion of cystic VS versus solid VS may explain their shorter tumour half-reduction time after GK radiosurgery.

Although the mechanism is not fully known, cystic expansion after radiosurgery in some cystic VS is acknowledged. ${ }^{56}$ Recent studies have suggested that, in cystic VS, GK radiosurgery could promote the haemorrhages that result in cyst

Table 2 Investigated factors related to tumour half-reduction time

\begin{tabular}{|c|c|c|c|c|c|}
\hline \multirow[b]{2}{*}{ Factor } & \multirow[b]{2}{*}{ Patient (number) } & \multirow{2}{*}{$\begin{array}{l}\text { Tumour half-reduction } \\
\text { time (mean, year) }\end{array}$} & \multirow[b]{2}{*}{ SD } & \multicolumn{2}{|l|}{$\mathrm{p}$ Value } \\
\hline & & & & Univariate & Multivariate \\
\hline \multicolumn{6}{|l|}{ Sex } \\
\hline Male & 18 & 3.5272 & 2.2757 & & \\
\hline \multicolumn{6}{|l|}{ Age } \\
\hline$\leqslant 40$ years & 20 & 9.0020 & 16.0154 & 0.679 & \\
\hline$\leqslant 3 \mathrm{ml}$ & 25 & 8.6016 & 19.8786 & 0.540 & \\
\hline$>3 \mathrm{ml}$ & 25 & 8.8048 & 12.0967 & & \\
\hline \multicolumn{6}{|l|}{ Marginal dose } \\
\hline$<12.5 \mathrm{~Gy}$ & 23 & 9.1348 & 14.8818 & 0.863 & \\
\hline$\geqslant 12.5 \mathrm{~Gy}$ & 27 & 8.3348 & 17.6679 & & \\
\hline \multicolumn{6}{|c|}{ Intratumoral necrosis } \\
\hline No & 33 & 12.0388 & 19.2778 & & \\
\hline \multicolumn{6}{|c|}{ Enhancement pattern } \\
\hline Dense & 28 & 8.8143 & 18.9460 & 0.957 & \\
\hline Sparse & 22 & 8.5609 & 14.5428 & & \\
\hline \multicolumn{6}{|l|}{ Tumour nature } \\
\hline Cystic & 24 & 2.5883 & 1.5585 & 0.009 & 0.006 \\
\hline Solid & 26 & 14.3469 & 21.1800 & & \\
\hline \multicolumn{6}{|l|}{ Tumour contour } \\
\hline Lobulating & 13 & 5.3577 & 6.1968 & 0.202 & \\
\hline Round & 37 & 9.8781 & 18.5134 & & \\
\hline
\end{tabular}

Continuous variables were dichotomised on the basis of their median values and variables' clinical significance. 
expansion. ${ }^{8}{ }^{23}$ We removed the cystic portion of cystic VS as much as possible during operation, and then treated the remaining solid portion of cystic VS using GK radiosurgery, which is why cyst expansion did not occur after GK radiosurgery in the present study.

In conclusion, GK radiosurgery appeared to be an effective treatment modality for residual tumour control after microsurgery. Owing to having relatively abundant tumour vascularity, residual solid portions of cystic VS resulted in efficient shrinkage after GK radiosurgery. Therefore, GK radiosurgery was found to be a rewarding therapeutic approach to the residual solid portions of cystic VS.

Competing interests: None.

\section{REFERENCES}

1. Kondziolka D, Lunsford LD, McLaughlin MR, et al. Long-term outcomes after radiosurgery for acoustic neuromas. N Engl J Med 1998;339:1426-33.

2. Flickinger JC, Kondziolka D, Niranjan A, et al. Results of acoustic neuroma radiosurgery: an analysis of 5 years' experience using current methods. $J$ Neurosurg 2001:94:1-6.

3. Lunsford LD, Niranjan A, Flickinger JC, et al. Radiosurgery of vestibular schwannomas: summary of experience in 829 cases. J Neurosurg 2005;102(Suppl):195-9.

4. Wowra B, Muacevic A, Jess-Hempen A, et al. Outpatient gamma knife surgery for vestibular schwannoma: definition of the therapeutic profile based on a 10-year experience. J Neurosurg 2005;102(Suppl):114-8.

5. Delsanti C, Regis J. Cystic vestibular schwannomas. Neurochirurgie 2004;50:4016.

6. Pendl G, Ganz JC, Kitz K, et al. Acoustic neurinomas with macrocysts treated with gamma knife radiosurgery. Stereotact Funct Neurosurg 1996;66(Suppl 1):103-11.

7. Flickinger JC, Kondziolka D, Pollock BE, et al. Evolution in technique for vestibular schwannoma radiosurgery and effect on outcome. Int J Radiat Oncol Biol Phys 1996;36:275-80
8. Park CK, Kim DC, Park SH, et al. Microhemorrhage, a possible mechanism for cyst formation in vestibular schwannomas. J Neurosurg 2006;105:576-80.

9. Gardner G, Robertson $\mathrm{JH}$. Hearing preservation in unilateral acoustic neuroma surgery. Ann Otol Rhinol Laryngol 1988:97:55-66.

10. House JW, Brackmann DE. Facial nerve grading system. Otolaryngol Head Neck Surg 1985;93:146-7.

11. Kondziolka D, Lunsford LD, Flickinger JC. The radiobiology of radiosurgery. Neurosurg Clin N Am 1999;10:157-66.

12. Linskey ME, Martinez AJ, Kondziolka D, et al. The radiobiology of human acoustic schwannoma xenografts after stereotactic radiosurgery evaluated in the subrenal capsule of athymic mice. J Neurosurg 1993;78:645-53.

13. Kamiryo T, Lopes MB, Kassell NF, et al. Radiosurgery-induced microvascular alterations precede necrosis of the brain neuropil. Neurosurgery 2001;49:409-14.

14. Niranjan A, Gobbel GT, Kondziolka D, et al. Experimental radiobiological investigations into radiosurgery: present understanding and future directions. Neurosurgery 2004;55:495-504.

15. Yamamoto M, Jimbo M, Kobayashi M, et al. Long-term results of radiosurgery for arteriovenous malformation: neurodiagnostic imaging and histological studies of angiographically confirmed nidus obliteration. Surg Neurol 1992;37:219-30.

16. Asano K, Ebina K, Sekiya $T$, et al. Acoustic neurinomas with large cystic components: a clinical and pathological study of 3 cases. No Shinkei Geka 1995;23:1075-82.

17. Sugihara S, Kinoshita T, Matsusue E, et al. Multicystic acoustic schwannoma with intratumoral hemorrhage: a report of two cases. Magn Reson Med Sci 2004;3:1014

18. Charabi S, Klinken L, Tos M, et al. Histopathology and growth pattern of cystic acoustic neuromas. Laryngoscope 1994;104:1348-52.

19. Wandong S, Meng L, Xingang L, et al. Cystic acoustic neuroma. J Clin Neurosci 2005:12:253-5

20. Muzumdar DP, Goel A, Pakhmode CK. Multicystic acoustic neurinoma: report of two cases. J Clin Neurosci 2002;9:453-5.

21. Shirato H, Sakamoto T, Takeichi N, et al. Fractionated stereotactic radiotherapy for vestibular schwannoma (VS): comparison between cystic-type and solid-type VS Int J Radiat Oncol Biol Phys 2000:48:1395-401.

22. Charabi S, Klinken L, Mantoni M, et al. Histology and neuro-imaging in cystic acoustic neuromas. Acta Otolaryngol 1993;113:519-23.

23. Suzuki H, Toyoda S, Muramatsu M, et al. Spontaneous haemorrhage into metastatic brain tumours after stereotactic radiosurgery using a linear accelerator. J Neurol Neurosurg Psychiatry 2003;74:908-12. 